Neue S3-Leitlinie zur Behandlung der Alkoholabhängigkeit

\title{
Wie Sie Ihren Patienten die ersten Schritte zur Abstinenz erleichtern
}

\author{
Alkoholkranke müssen nicht unbedingt abrupt mit dem Trinken aufhören, auch weniger Alkohol \\ kann ein wichtiges Zwischenziel sein.
}

In Deutschland sterben jedes Jahr etwa 74.000 Menschen an den Folgen eines schädlichen Alkoholkonsums. Ein großes Problem ist nach wie vor, dass viele Suchtkranke keine ärztliche Hilfe in Anspruch nehmen.

In der Leitlinie „Screening, Diagnose und Behandlung alkoholbezogener Störungen" wird erstmals auch eine Reduktion des Alkoholkonsums bei Patienten mit Alkoholmissbrauch als wichtiges Therapieziel genannt. Begründet wird dies mit Studien, nach denen nur etwa die Hälfte der behandlungsbedürftigen Personen mit Alkoholproblemen „trotz Einsicht in die Notwendigkeit einer Behandlung nicht oder noch nicht bereit war, vollständig auf Alkohol zu verzichten“, heißt es in der Leitlinie. Bereits 2011 kam die entsprechende britische Therapieleitlinie des NICE zu dem Schluss, auch eine Reduktion der Trinkmengen als intermediäres Therapieziel für Alkoholabhängige

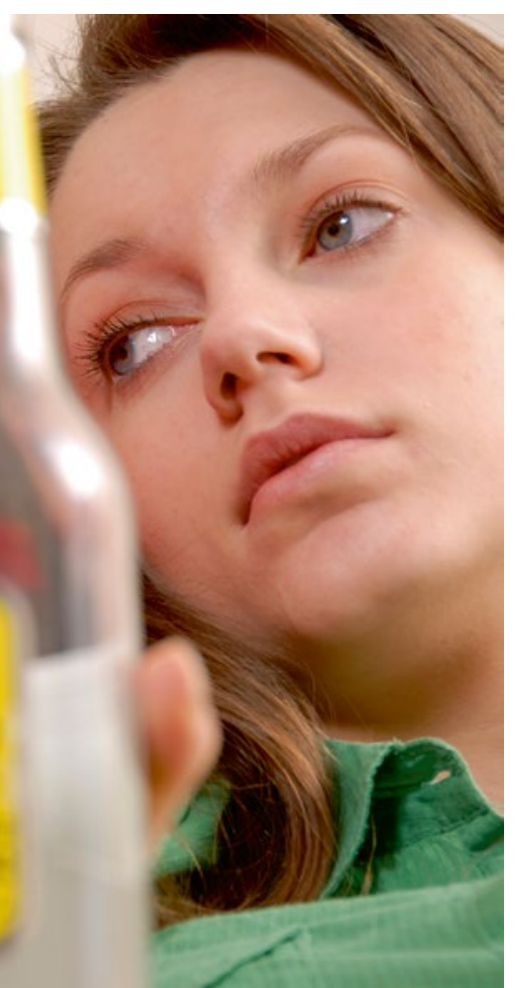

anzuerkennen. Diesen Standpunkt vertritt inzwischen auch die EMA. „Wir hoffen, dass die damit verbundene Senkung der Eingangsschwellen deutlich mehr Menschen in eine Beratung und Behandlung führt als bisher. Könnte die Inanspruchnahme von psycho- und pharmakotherapeutischen Angeboten von bisher $10 \%$ auf $40 \%$ der Betroffenen erhöht werden, ließen sich nach einer aktuellen Modellrechnung pro Jahr rund 2.000 Menschenleben in Deutschland retten“, schreiben die Leitlinienautoren um Prof. Karl Mann vom Zentralinstitut für Seelische Gesundheit in Mannheim.

\section{AUDIT-Fragebogen zum Screening}

Ein Schwerpunkt bei der Diagnostik von Alkoholstörungen bildet das Screening per Fragebogen. Die Leitlinie rät zum Alcohol Use Disorders Identification Test (AUDIT) oder, wenn für die zehn Fragen des AUDIT keine ausreichende
Zeit zur Verfügung steht, zur Kurzversion mit drei Fragen (AUDIT-C). In der Kurzversion wird nach der Häufigkeit des Alkoholkonsums in Tagen pro Woche, nach der durchschnittlichen Zahl der konsumierten alkoholischen Getränke und nach Tagen mit extrem hohem Konsum gefragt.

Bei der Therapie von Alkoholkranken soll eine medikamentenunterstützte qualifizierte Entzugsbehandlung statt einer reinen körperlichen Entgiftung angeboten werden. Um die Schwere und Häufigkeit von Alkoholentzugssymptomen sowie Entzugskomplikationen wie Delire und Krampfanfälle zu vermeiden, werden primär Benzodiazepine empfohlen (Empfehlungsgrad A).

\section{Thomas Müller}

- S3-Leitlinie „Screening, Diagnose und Behandlung alkoholbezogener Störungen"

http://www.dgppn.de/fileadmin/user_upload/_medien/ download/pdf/kurzversion-leitlinien/S3-Leitlinie_Alkohol_2015_Langfassung.pdf

\section{Neue Zahlen zum Alkoholkonsum Jugendlicher}

Einer aktuellen Erhebung der Bundeszentrale für gesundheitliche Aufklärung BZgA zufolge machen mehr als zwei Drittel der Jugendlichen erste Erfahrungen mit dem Alkohol im Alter von knapp 14 Jahren, und damit fast ein Jahr später als noch zehn Jahre zuvor. Der Anteil der 12- bis 17-Jährigen, die innerhalb der letzten zwölf Monate mindestens einmal pro Woche Alkohol getrunken haben, liegt bei Jungen bei $18 \%$ und bei Mädchen bei $9 \%$.

Rauschtrinken ( $\geq$ sechs alkoholische Getränke bei einer Gelegenheit, mindestens einmal im Monat) schlägt besonders in der Altersgruppe der 14- bis 17-Jährigen zu Buche. 23,2\% der Jungen und 16,2\% der Mädchen setzen sich regelmäßig einem exzessiven Alkoholrausch aus (KiGGS Welle-1). Auffällig ist dabei die abweichende Selbsteinschätzung: Mehr als die Hälfte der jugendlichen Rauschtrinker war nach eigener Auffassung gar nicht betrunken.

Insgesamt zeichnet sich aber ab, dass heute weniger Jugendliche Alkohol trinken als noch vor einigen Jahren. So ist der Anteil der 11- bis 17-Jährigen, die jemals Alkohol getrunken haben, seit den Jahren 2003-2006 bis zum Zeitraum 2009-2012 von 62,8\% auf 54,4\% gesunken.

Deutlich wird diese Entwicklung auch anhand der Berichte der Notfallstationen: Erstmals konnte im Jahr 2013 ein Rückgang der alkoholbedingten stationären Aufnahmen von Jugendlichen um rund $13 \%$ gegenüber dem Vorjahr verzeichnet werden. 